\title{
Study of Thermal Effects Induced by a Laser Radiation Incident in an Amplifier (II): Nonlinear Case
}

\author{
M. M. El-Nicklawy, A. F. Hasssan, S. El-Din Salman, S. L. Diab \\ and A. M Abd-Rabou
}

Physics Department, Faculty of Science, Helwan University

The present work deals with the study of the temperature distribution generated in a ruby rod resulting from (i) a pump radiation incident in radial direction and (ii) the laser radiation incident in axial direction. The pump radiation originated from a source located in one focal axis of a cylinder having an elliptical cross section while the ruby rod is mounted in the other focal axis. The study includes the effect of the focusing parameter of the pump radiation, the half width of the incident laser beam which is assumed to have a radial Gaussian intensity distribution and the saturation of the net amplification coefficient resulting from the laser beam. The results show that by decreasing the focusing of the pump radiation, the radial temperature distribution resulting from the laser beam exhibits a dip and that the net amplification coefficient grows stepwise as $r$ increases.

\section{Introduction:}

Since the discovering of stimulated emission, the research in many developing centers aimed to increase the emitting power of the laser radiation to be applied in machining processing. This was necessary because of the tiny focusing radius of the laser beam which allows fine details to be processed in materials such as welding, cutting and drilling of holes [1-3]. Sabaeian et al. [4] have investigated the effects of heat load on longitudinal diode pumped solid state laser for generation of Bessel-Gaussian beam under Gaussian and supper Gaussian pump profiles. Van Elsen et al. [5] gave an analytical and numerical solution of the heat conduction solution for localized moving heat source in laser material processing. Advanced material processing with nanosecond and femtosecond pulsed laser was given by Kurita et al. [6]. A numerical simulation of machining glass by dual $\mathrm{Co}_{2}$ - laser beams was investigated by Jiao et al. [7]. Moreover high power lasers can be applied in plasma generation, changing the phase of absorbing material [8] and producing p-n junction [9]. During the 
research process the investigators were faced with the increase of the temperature resulting from the pump process, the stimulated intensity within the oscillator and the amplified intensity during the propagation of the beam in an amplifying medium. Due to this fact, thermal stresses as well as variation of refractive index and curvature of the end faces of solid laser rods led to damage the laser material or to strong variation of the front surface of the laser beam. The latter led to an inaccurate laser machining.

To investigate temperature distribution within the lasing material several authors have tried to solve this problem either analytically or numerically in different laser media so for instance have $[10,11]$ studied the effect of the temperature on the creation of variation in the refractive index as well as mechanical deformation, stresses and changes resulting from lensing aberration in the laser cavity. Marcello et al. [12] gave an analytical method for thermal lay out optimization of multilayer structure solid state devices.

This paper aims to study the special and temporal temperature distribution within a solid lasing rod resulting from a pump beam and a strong laser radiation to be amplified during its propagation in the lasing material. The study is devoted to account for saturated nonlinearity in the amplification coefficient resulting from the laser beam to be amplified.

\section{Theory}

Assuming a homogenous isotropic medium in the form of rod with circular cross section to be air cooled and pumped radially at its outer surface with a radiation originating from a black body source. The source and the rod were located in the two focal axis of a cylinder having elliptical cross section and reflecting outer surface. The calculated temporal profiles will result from both the pump source and the laser beam to be amplified which are considered to have a Ready distribution [13] with coinciding maxima. Since both pulses are of different pulse durations, the on set of the laser pulse was temporally retarded.

The spatial distribution of the laser radiation, which was amplified through out its propagation along the axis of the amplifier, was considered to have either a Gaussian shaped radial distribution of different constant or increasing cross section along the propagation direction.

To get the temperature distribution within the target, the following set of equations is considered:

(1) The heat diffusion equation in cylindrical coordinates assuming rotational system: 
(i) Originating from the pump beam:

$$
\frac{\partial^{2} T_{p}(r, t)}{\partial r^{2}}+\frac{1}{r} \frac{\partial T_{p}(r, t)}{\partial r}+\frac{1}{k} g_{p}(r, t)=\frac{1}{\alpha} \frac{\partial T_{p}(r, t)}{\partial t}
$$

where:

$T_{p}(r, t)$ is the temperature distribution generated from the absorbed pump radiation,

$g_{p}(r, t)$ is the rate of energy generated from the absorbed part of the pump beam per unit volume,

$\alpha=\frac{k}{\rho C_{p}}$ is the thermal diffusivity of the amplifier's material,

$k$ is the thermal conductivity of the amplifier's material,

$\rho$ is the mass density of the amplifier's material and

$C_{p}$ is the specific heat of the amplifier's material at constant pressure.

(ii) Originating from the laser radiation:

$\frac{\partial^{2} T_{L}(r, z, t)}{\partial r^{2}}+\frac{1}{r} \frac{\partial T_{L}(r, z, t)}{\partial r}+\frac{\partial^{2} T_{L}(r, z, t)}{\partial z^{2}}+\frac{1}{k} g_{L}(r, z, t)=\frac{1}{\alpha} \frac{\partial T_{L}(r, z, t)}{\partial t}$

where:

$T_{L}(r, z, t)$ is the temperature distribution generated from the absorbed part of the laser beam to be amplified and

$g_{L}(r, z, t)$ is the rate of energy generated from the absorbed part of the amplified laser radiation per unit volume,

(2) The boundary condition describing the cooling at the outer radius of the amplifying rod subjected to the pump process and the laser radiation to be amplified:

$$
-\left.k \frac{\partial T_{t}(r, z, t)}{\partial r}\right|_{r=R}=\left.h T_{t}(r, z, t)\right|_{r=R}
$$

where:

$R \quad$ is the radius of the rod,

$h \quad$ is the forced convection heat transfer coefficient and

$T_{t}(r, z, t)$ is the total temperature distribution given by equation (4).

Since the system of the equations governing the temperature distribution is a linear one, therefore the total temperature distribution will be given by

$$
T_{t}(r, z, t)=T_{L}(r, z, t)+T_{p}(r, t)
$$


The temperature distribution resulting from the pump process was obtained from the Green's function which results from the solution of the homogenous heat diffusion equation subjected to an initial condition. From the obtained Green's function the temperature distribution can be generally calculated from the following equation [14]:

$$
\begin{aligned}
T(r, t)= & \left.\int_{\bar{R}} r^{\prime} G\left(\vec{r}, t \mid \vec{r}^{\prime}, \tau\right)\right|_{\tau=0} F\left(\vec{r}^{\prime}\right) d V^{\prime}+\frac{\alpha}{k} \int_{\tau=0} d \tau \int_{\bar{R}} \vec{r}^{\prime} G\left(\vec{r}, t \mid \vec{r}^{\prime}, \tau\right) g\left(\vec{r}^{\prime}, \tau\right) d V^{\prime} \\
& +\left.\alpha \int_{\tau=0}^{t} d \tau \sum_{i=1 S_{i}}^{N} \vec{r}^{\prime} G\left(\vec{r}, t \mid \vec{r}^{\prime}, \tau\right)\right|_{r^{\prime}=r_{i}} \frac{1}{k} f_{i}\left(\vec{r}^{\prime}, \tau\right) d S_{i}^{\prime}
\end{aligned}
$$

where:

$\bar{R} \quad$ is the entire volume of the considered region,

$S_{i} \quad$ is the boundary surface of region $R, i=1,2,3, \ldots, N$,

$N \quad$ is the number of continuous surfaces,

$d V^{\prime}$ and $d S_{i}^{\prime}$ are the differential volume and surface element in the $\vec{r}^{\prime}$ variable,

respectively.

$F\left(\vec{r}^{\prime}\right) \quad$ is the initial temperature distribution,

$g(\vec{r}, t) \quad$ represents the contribution of the energy generation leading to heat

$G\left(\vec{r}, t \mid \vec{r}^{\prime}, \tau\right)$ represents the Green's function,

$f_{i}(\vec{r}, \tau) \quad$ represents the non-homogenous terms of the boundary condition.

Setting $F\left(\vec{r}^{\prime}\right)$ and $f_{i}(\vec{r}, \tau)$ equal to zero, one gets

$$
\left.G_{p}\left(\vec{r}, t \mid \vec{r}^{\prime}, \tau\right)\right|_{\tau=0}=\sum_{m=1}^{\infty} e^{-\alpha \beta_{m}^{2} t} \frac{1}{N\left(\beta_{m}\right)} J_{\circ}\left(\beta_{m} r\right) J_{\circ}\left(\beta_{m} r^{\prime}\right)
$$

where:

$\beta_{m} \quad$ are the eigenvalues of the solution of the heat diffusion equation,

$N\left(\beta_{m}\right)$ is the norm factor and

$J_{\circ}\left(\beta_{m} r\right)$ is the Bessel function of zero order and first kind,

The temperature distribution resulting from the pump source can, thus, be given by

$$
T_{p}(r, t)=\frac{\alpha}{k} \int_{\tau=0} d \tau \int_{\bar{R}} r^{\prime} G_{p}\left(r, t \mid r^{\prime}, \tau\right) g_{p}\left(r^{\prime}, \tau\right) d V^{\prime}
$$

with $g_{p}(r, t)=g_{p}(r) G_{p}(t)$ and $g_{p}(r)=\frac{R_{\circ}+R}{R_{\circ}+r} e^{\tilde{\alpha}(v)(r-R)}$

to be determined later and 


$$
g\left(\vec{r}^{\prime}, t\right)=g_{p}\left(\vec{r}^{\prime}, t\right)=X \int_{v=0}^{\infty} \tilde{\alpha}(v) G_{p}(t) I_{\text {opv }}(v) \frac{R_{\circ}+R}{R_{\circ}+r} e^{\tilde{\alpha}(v)(r-R)} d v
$$

where:

$\tilde{\alpha}(v)$ is the intensity independent attenuation coefficient of the rod at the frequency $v$,

$R_{\circ} \quad$ is the radius of the focused pump radiation,

$X \quad$ represents the part of the absorbed radiation which heats the rod and

$I_{\text {opv }}(v)$ is the spectral intensity distribution at frequency $v$ of the black body representing the pump radiation.

The temperature resulting from the absorbed laser radiation is found, after applying the two dimensional Laplace integral transform technique, to be given by

$$
\begin{aligned}
T_{L}(r, z, t)= & \frac{2 \pi \alpha}{k} \int_{r=0}^{R} \int_{\tau=0}^{t} r^{\prime} \sum_{m=0}^{\infty} \frac{2 \beta_{m}^{2}}{R^{2}\left(H^{2}+\beta_{m}^{2}\right)} \cdot \frac{1}{J_{\circ}^{2}\left(\beta_{m} R\right)} e^{-\alpha \beta_{m}^{2}(t-\tau)} \\
& J_{\circ}\left(\beta_{m} r^{\prime}\right) J_{\circ}\left(\beta_{m} r\right) \int_{0}^{L} G_{L}\left(Z, t \mid z^{\prime}, \tau\right) g_{L}\left(r^{\prime}, z^{\prime}, \tau\right) d r^{\prime} d z^{\prime} d \tau
\end{aligned}
$$

where $H=\frac{h}{k}$ with

$$
\begin{aligned}
& G_{L}\left(z, t \mid z^{\prime}, \tau\right)_{\tau=0}=\frac{1}{2 \sqrt{\pi \alpha t}}\left[\exp \left[-\left(\frac{2 L-z-z^{\prime}}{2 \sqrt{\alpha}}\right)^{2} \frac{1}{t}\right]+\exp \left[-\left(\frac{z+z^{\prime}}{2 \sqrt{\alpha}}\right)^{2} \frac{1}{t}\right]+\right. \\
& \exp \left[-\left(\frac{2 L+z-z^{\prime}}{2 \sqrt{\alpha}}\right)^{2} \frac{1}{t}\right]+\exp \left[-\left(\frac{z-z^{\prime}}{2 \sqrt{\alpha}}\right)^{2} \frac{1}{t}\right]+\exp \left[-\left(\frac{2 L+z-z^{\prime}}{2 \sqrt{\alpha}}\right)^{2} \frac{1}{t}\right]+ \\
& \left.\exp \left[-\left(\frac{4 L-z-z^{\prime}}{2 \sqrt{\alpha}}\right)^{2} \frac{1}{t}\right]+\exp \left[-\left(\frac{2 L+z+z^{\prime}}{2 \sqrt{\alpha}}\right)^{2} \frac{1}{t}\right]+\exp \left[-\left(\frac{4 L+z-z^{\prime}}{2 \sqrt{\alpha}}\right)^{2} \frac{1}{t}\right]\right](10) \\
& g_{L}(r, z, t, v)=\int_{v} I_{o L v}(v, r, 0, t) \alpha_{h} \exp -\left(\alpha_{L}(v, r, t)-\beta_{L}(v, r, t)\right) z d v
\end{aligned}
$$

where:

$I_{o L v}(v, r, 0, t)$ is the spectral intensity distribution in interval $v \rightarrow v+d v$ of the laser beam incident in $z$ direction on the pumped laser rod at $z=0$, $\alpha_{L}(v, r, t) \quad$ is the linear attenuation coefficient of the pumped rod in the frequency range of the incident laser radiation, 
$\alpha_{h} \quad$ is the part of the linear attenuation coefficient responsible for the heating process and it is equals to $201 / \mathrm{m}$ [15] and

$\beta_{L}(v, r, t)$ is the linear amplification coefficient of the pumped rod in the frequency range of the incident laser radiation.

Equation (11) will be developed with

$$
I_{o L v}=\tilde{I}_{\circ} g_{L}\left(v, v_{o L}\right) g_{L}(r) G_{L}(t)
$$

and

$$
\begin{aligned}
& g_{L}\left(v, v_{o L}\right)=\frac{1}{\Delta v_{o L}} \sqrt{\frac{4 \ln 2}{\pi}} \exp \left(4 \ln 2\left(\frac{v-v_{o L}}{\Delta v_{o L}}\right)\right) \\
& \beta_{L}(v, r, t)-\alpha_{L}(v, r, t)=\frac{c^{2} A_{21}}{8 \pi v_{\circ}^{2}} g_{m}\left(v, v_{\circ}\right)\left(N_{2}-\frac{g_{2}}{g_{1}} N_{1}\right) \\
& g_{m}\left(v, v_{\circ}\right)=\frac{1}{\Delta v_{\circ}} \sqrt{\frac{4 \ln 2}{\pi}} \exp \left(-\left(\frac{v-v_{\circ}}{\Delta v_{\circ}}\right)^{2} 4 \ln 2\right) \\
& \left(N_{2}-\frac{g_{2}}{g_{1}} N_{1}\right)=\frac{1}{L}\left(n \ln 10 \sqrt{\frac{\pi}{4 \ln 2}} \frac{8 \pi v_{\circ}^{2} \Delta v_{\circ}}{c^{2} A_{21}}\right)
\end{aligned}
$$

where:

$g_{L}(r) \quad$ is the redial profile of the laser beam,

$G_{L}(t) \quad$ is the time profile of the laser pulse,

$g_{L}\left(v, v_{o L}\right)$ is the normalized spectral profile of the $(0,0)$ mode of the laser radiation,

$\Delta v_{o L} \quad$ is the full width at half maximum of $(0,0)$ mode laser radiation,

$v_{o L} \quad$ is the central frequency of the $(0,0)$ mode of the laser radiation,

$g_{m}\left(v, v_{\circ}\right)$ is the spontaneous spectral line profile of the medium,

$v$ o is the central frequency of the spontaneous emission of the medium,

$\Delta v_{0} \quad$ is the full width of the half maximum of the spontaneous emission of the medium,

$N_{1}$ and $N_{2}$ are the densities of the atoms in the lower and upper level respectively,

$g_{1}$ and $g_{2}$ are their statistical weights respectively and

$L \quad$ is the length of the amplifying rod. 
Equation (13) considers an amplification at the temporal maximum of the pump radiation $(t=\tilde{t})$ at the end of the amplifier $(\mathrm{z}=L)$ for $r=0$ and $R_{\circ}=10^{-3} \mathrm{~m}$ to be given by $e^{\left(\beta_{L}(v, r, \tilde{t})-\alpha_{L}(v, r, \tilde{t})\right) L}=10^{n}$. According to this requirement the spectral density of the pump radiation $\rho(v)$, necessary for initiating the required amplification in case of a three level system, is given by

$$
\rho(v)=\frac{A_{21}}{B_{13}} \frac{N_{\circ}+\frac{n \ln 10}{L} \sqrt{\frac{\pi}{4 \ln 2}} \frac{8 \pi v_{\circ}^{2} \Delta v_{\circ}}{c^{2} A_{21}}}{\frac{n \ln 10}{L} \sqrt{\frac{\pi}{4 \ln 2}} \frac{8 \pi v_{\circ}^{2} \Delta v_{\circ}}{c^{2} A_{21}}}
$$

$B_{13}$ can be obtained from the maximum of the linear absorption coefficient of the two absorbed lines $F_{1}^{4}$ and $F_{2}^{4}$ [15] for the cases of parallel and perpendicular incident radiation. Under these conditions the temperature of the pump radiation resulting from the black body is given by

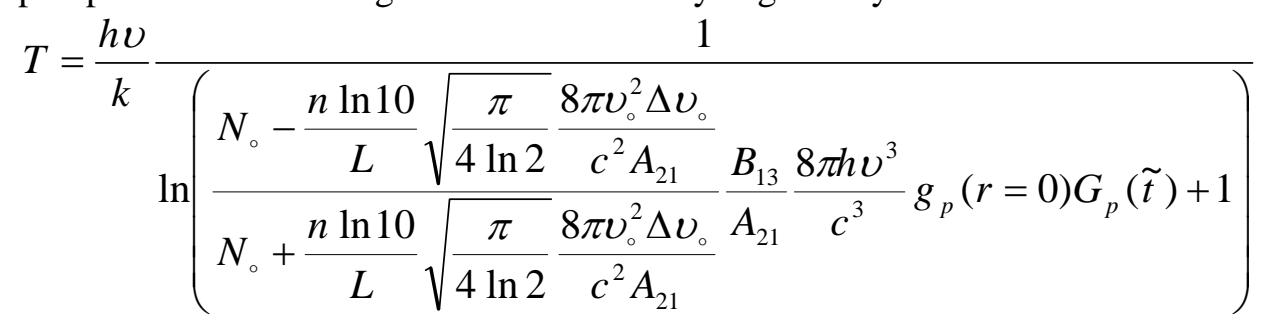

Thus,

$$
\beta_{L}\left(v_{o L}, r, L, t\right)-\alpha_{L}\left(v_{o L}, r, L, t\right)=\sqrt{\frac{4 \ln 2}{\pi}} \frac{1}{\Delta v_{o L}} \frac{c^{2} A_{21}}{8 \pi v_{\circ}^{2}} N_{\circ} \frac{\frac{B_{13}}{A_{21}} \rho(v) g_{p}(r) G_{p}(t)-1}{\frac{B_{13}}{A_{21}} \rho(v) g_{p}(r) G_{p}(t)+1}
$$

Determination of the heat generation function.

\section{(a) Resulting from the pump radiation $g_{p}(r, t)$.}

Due to the radiation diffraction from the reflecting cylinder surface and the imperfection of its elliptical cross section, circular spot of radius $R_{\circ}$ with its centre located on the focal axis of the cylinder will be considered.

This assumptions leads to the following differential equation for the intensity distribution in radial direction within the rod:

$$
\frac{d I_{p}(v, r)}{d r}=-\frac{I_{p}(v, r)}{R_{\circ}+r}+\tilde{\alpha}(v) I_{p}(v, r)
$$


The solution of Eqn. (17) gives with the boundary condition $I_{p}(v, R)=I_{o p}(v)$

$I_{p}(v, r)=I_{o p}(v) \frac{R_{\circ}+R}{R_{\circ}+r} e^{\tilde{\alpha}(v)(r-R)}$

Since the pump radiation is considered to be that of a black body and the absorption spectrum of the rod is broad, therefore $I_{p}(v, r)$ and $I_{o p}(v)$ will be replaced by $I_{p v}(v, r) d v$ and $I_{o p v}(v) d v$, respectively.

The generation function responsible for the heating process can be obtained from the first derivative of the exponential factor with respect to $r$ (modified first derivatives), which gives

$\int I_{\text {opv }}(v) d v=g_{p}(r, t)=\int_{v_{1}}^{v_{2}} I_{\text {opv }}(v) \tilde{\alpha}(v) \frac{R_{\circ}+R}{R_{\circ}+r} e^{\tilde{\alpha}(v)(r-R)} d v$

where $\tilde{\alpha}(v)$ is the intensity independent linear attenuation coefficient of the rod at the frequency $v$.

(b) Resulting from the laser beam to be amplified through the pumped rod $g_{L}(r, z, t)$.

Because of the strong intensity distribution of the laser radiation the net amplification factor resulting from the absorbed and amplified parts cannot be considered as independent of the laser radiation. To get a mathematical expression for its dependence on the laser radiation, a homogenous brooded atomic system in equilibrium in the absence of external radiation field is considered. The rates $R_{1}$ and $R_{2}$ at which atoms are feed into these levels might be balanced by spontaneous and radiative loss processes such as collisions. This gives for the case of equal degeneracy of the considered levels

$$
N_{2}-N_{1}=\Delta \stackrel{\circ}{N}=R_{2} \tau_{2}-\tau_{1}\left(R_{1}+R_{2} \tau_{2} A_{21}\right)
$$

where $\tau_{1}$ and $\tau_{2}$ are the effective life times resulting from spontaneous and nonradiative processes for the lower and upper levels, respectively. $A_{21}$ is Einstein coefficient for spontaneous emission from level two to level one. If a monochromatic or other signal is fed into the system then stimulated absorption and emission will occur. Under this considerations, $\left(N_{2}-N_{1}\right)$ for the two energy levels under equilibrium is given by

$$
N_{2}-N_{1}=\frac{\Delta \stackrel{\circ}{N}}{1+\varphi_{v} \omega / A_{21}}
$$


with $\omega=\frac{c^{2} A_{21}}{g \pi h v^{3}} I(v) g\left(v_{\circ}, v\right)$,

$$
\varphi_{v}=A_{21} \tau_{2}\left(1+\left(1-A_{21} \tau_{2}\right) \frac{\tau_{1}}{\tau_{2}}\right)
$$

and $I_{s}(v)=\frac{8 \pi h v^{3}}{c^{2} \varphi_{v} g\left(v_{\circ}, v\right)}$

Thus the gain as a function of the intensity in a homogenous broadened system is given by $\gamma(v)=\frac{\Delta \stackrel{\circ}{N}}{1+I(v) / I_{s}(v)} \frac{c^{2} A_{21}}{8 \pi h v^{2}} g(v, v)$

where

$g\left(v_{0}, v\right)$ is the line profile of the transition of the considered atoms,

$v$ o is the central frequency and

$I(v)$ is the spectral intensity of the field leading to stimulated emission and absorption.

Considering this behaviour in the net amplification factor which is given by $\left(N_{2}-N_{1}\right) \frac{c^{2} A_{21}}{8 \pi h v^{2}} \tilde{g}_{L}\left(v, v_{\circ}\right)$ one gets after substituting for $N_{2}-N_{1}$ from

Eqn. (20)

$$
\left(\beta_{L}(v, r, z, t)-\alpha_{L}(v, r, z, t)\right)_{N L}=\frac{\left(\beta_{L}(v, r, t)-\alpha_{L}(v, r, t)\right)_{L}}{1+g_{L}(r) g_{L}(t) \frac{I(z, v)}{I_{s}}}
$$

To get $I(z, v)$, one has to solve the differential equation

$$
\frac{d I(v, r, z, t)}{d z}=\frac{\gamma_{\circ}}{1+\frac{I(v, r, z, t)}{I_{s}}} I(v, r, z, t)
$$

with $I(v, r, z, t)=I(z) G_{L}(t) g_{L}(r) g_{L}\left(v, v_{\circ}\right)$

and

$\gamma_{\circ}=\Delta \stackrel{\circ}{N} \frac{c^{2} A_{21}}{8 \pi h v^{2}} \tilde{g}_{L}\left(v, v_{\circ}\right)=\beta_{L}\left(v_{o L}, r, L, t\right)-\alpha_{L}\left(v_{o L}, r, L, t\right)$

where $G_{L}(t), g_{L}(r)$ and $g_{L}\left(v, v_{\circ}\right)$ is the temporal, radial and spectral distribution of the laser pulse, respectively. 
This gives under the boundary condition at $z=0$ :

$$
\begin{aligned}
I(v, r, z=0, t) & =\hat{I}_{\circ} G_{L}(t) g_{L}(r) g_{L}\left(v, v_{\circ}\right) \\
I(v, r, z, t) & =\hat{I}_{\circ} \exp \left(\gamma_{\circ} z+\frac{1}{I_{s}}\left(\hat{I}_{\circ}-I(v, r, z, t)\right)\right)
\end{aligned}
$$

Thus, $\frac{d I(v, r, z, t)}{d z}$ initiating the heating process can be given by

$$
\frac{d I(v, r, z . t)}{d z}=\frac{\alpha_{L}\left(v, v_{\circ}\right) \hat{I}_{\circ}}{1+\frac{\hat{I}_{\circ}}{I_{s}}} \exp \left(\gamma_{\circ} z+\frac{1}{I_{s}}\left(\hat{I}_{\circ}-I(v, r, z, t)\right)\right)
$$

Since Newton and other iteration methods [16] are converging very slowly, therefore the average value of the intensity within small slices along $z$ direction of the amplifier has to be calculated and replaced for $I(v, r, z, t)$ in equation (24) giving an average value for $\beta_{L}(v, r, z, t)-\alpha_{L}(v, r, z, t)$ within the interval $z^{\prime}$ to $z^{\prime}+\Delta z^{\prime}$. Replacing this average value for $\beta_{L}(v, r, z, t)-\alpha_{L}(v, r, z, t)$ in the equation of the heat generation function given by:

$$
g_{L}(r, z, t, v)=\int_{v} I_{o L v}(v, r, 0, t) \alpha_{L} \exp -\left(\alpha_{L}(v, r, t)-\beta_{L}(v, r, t)\right) z d v,
$$

one gets the source of heating originating from the laser radiation $g_{L}(v, r, z, t)$ which is necessary for the solution of Eqn. (2).

\section{Computation.}

The temperature distribution, resulting from Eqn. (4) after replacing for $T_{p}(r, t)$ and $\mathrm{T}_{\mathrm{L}}(\mathrm{r}, \mathrm{z}, \mathrm{t})$ from Eqns. (7 and 9), respectively, was calculated for a spatial Gaussian distributed laser intensity having a constant half width $w_{\circ}$ at which $I_{\circ}$ reduces to $\frac{I_{\circ}}{e^{2}}$.

$$
I=I_{\circ} \exp \left(\frac{-2 r^{2}}{w_{\circ}^{2}}\right)
$$

The time dependence for the pump beam and the laser beam are given [13] respectively by: 


$$
\begin{aligned}
& G_{p}(t)=\frac{(n+1)^{n+1}}{n^{n}} \cdot \frac{t}{\Delta t_{p}} \cdot\left(1-\frac{t}{\Delta t_{p}}\right)^{n} \quad n=3 \quad 0 \leq t \leq \Delta t_{p} \\
& G_{L}(t)=\frac{(n+1)^{n+1}}{n^{n}} \cdot \frac{\left(t-\Delta t_{\mathrm{o}}\right)}{\Delta t_{L}} \cdot\left(1-\frac{\left(t-\Delta t_{\mathrm{o}}\right)}{\Delta t_{L}}\right)^{n} \quad n=3 \quad 0 \leq t \leq \Delta t_{L}
\end{aligned}
$$

$\Delta t_{L}$ and $\Delta t_{p}$ are the pulse duration of the laser and pump pulse respectively and $\Delta t$ is the time retardation leading to coinciding the two maxima.

The calculation was carried out for $w_{\circ}=10^{-4}, 5 \times 10^{-4}$ and $10^{-3} \mathrm{~m}$.

Since the laser radiation was assumed to be un-polarized with equal probability of parallel and perpendicular components, $\tilde{\alpha}(v)$ was calculated from the summation of the values of the parallel and perpendicular polarized components given in [15]. The physical and thermal parameters required for the computation of the temperature distribution in Ruby laser are given according to [15] in Table (1).

\begin{tabular}{|c|c|c|c|c|c|}
\hline Symbol & $\lambda(m)$ & $\rho\left(\mathrm{kg} / \mathrm{m}^{3}\right)$ & $C_{\mathrm{p}}\left(\right.$ W.s. $\left./ \mathrm{kg} .{ }^{\circ} \mathrm{K}\right)$ & $N_{\circ}\left(\right.$ ions $\left./ m_{3}\right)$ & $v_{01}(\mathrm{~Hz})$ \\
\hline Value & $6943 \times 10^{-10}$ & 4300 & 43 & $1.58 \times 10^{25}$ & $1.5 \times 10^{15}$ \\
\hline Symbol & $v_{21}(\mathrm{~Hz})$ & $v_{22}(\mathrm{~Hz})$ & $v_{31}(\mathrm{~Hz})$ & $v_{32}(\mathrm{~Hz})$ & $F_{\perp}^{21}(1 / m)$ \\
\hline Value & $7.3 \times 10^{14}$ & $7.1 \times 10^{14}$ & $5.4 \times 10^{14}$ & $5.53 \times 10^{14}$ & 320 \\
\hline Symbol & $F_{\perp}^{31}(1 / m)$ & $F_{I I}^{32}(1 / m)$ & $F_{I I}^{01}(1 / m)$ & $F_{I I}^{22}(1 / m)$ & $\Delta v_{\circ}(H z)$ \\
\hline Value & 155 & 285 & 360 & 280 & $0.329 \times 10^{12}$ \\
\hline Symbol & $\begin{array}{c}k \\
\left(W / m^{\circ} K\right)\end{array}$ & $\begin{array}{c}h \\
\left(W / m^{2} .{ }^{\circ} K\right)\end{array}$ & & & \\
\hline Value & 42 & $1 \times 10^{5}$ & & & \\
\hline
\end{tabular}

Table (1)

\section{Results and Discussion.}

\section{(a) Heating resulting from pump radiation.}

Figure (1) represents the time dependence of the temperature induced by the pump radiation for an arbitrary $\mathrm{z}$ value, $r=0$ and $R_{\circ}$ as a parameter. From the figure it is evident that the maximum temperature occurs at times greater than $t=\tilde{t}$ which is due to the fact that, up to this time the absorbed radiation overcompensates the heat conduction inside the cooler part of the rod. 
Moreover, it is observed that the maximum temperature decreases with increasing $R_{\circ}$ value which matches the fact of the decrease of the intensity with increasing $R_{\circ}$. The appearance of a relative maximum at much greater times than $t=\tilde{t}$ is due to the conduction of the heat generation resulting from the outer surface of the rod which is more pronounced as $R_{\circ}$ increases and which needs relatively great times to reach the axial point.

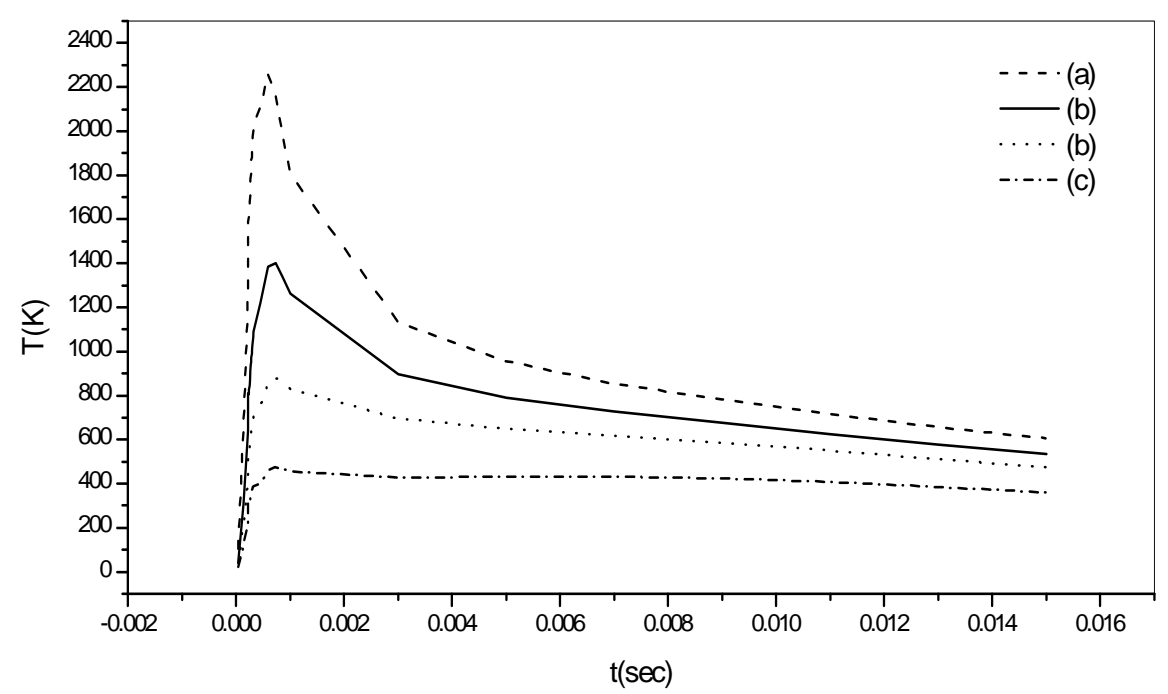

Fig. (1): The temporal temperature distribution induced by the pump radiation calculated for $r=0$ : (a) $R_{\circ}=10^{-4} \mathrm{~m}$, (b) $\mathrm{R}_{\circ}=5 \times 10^{-4} \mathrm{~m}, \quad$ (c) $\mathrm{R}_{\circ}=10^{-3} \mathrm{~m}$ and (d) $\mathrm{R}_{\mathrm{o}}=5 \times 10^{-3} \mathrm{~m}$.

Figure (2) represents the radial temperature distribution calculated for any arbitrary $\mathrm{z}$ at $t=\tilde{t}$ and $R_{\circ}$ as a parameter. The curves show a relative maximum at $r=0$ this is due to the higher intensity compared with its near surrounding. The decrease of the maximum temperature at $r=0$ and its nearby surrounding with increasing $R_{\text {。 }}$ values is due to the variation of the intensity resulting from focusing. The temperature remains at $r=R$ practically constant. This behaviour is due to the radiation intensity distribution which is in this case equals to $I=I_{o}$. Due to the decrease of the radiation at $r=0$ by increasing $R_{\text {。 }}$ values ant its constancy at $R=r$, the temperature at $r=R$ attains under these circumstances values which are comparable or greater than that at $r=0$. 


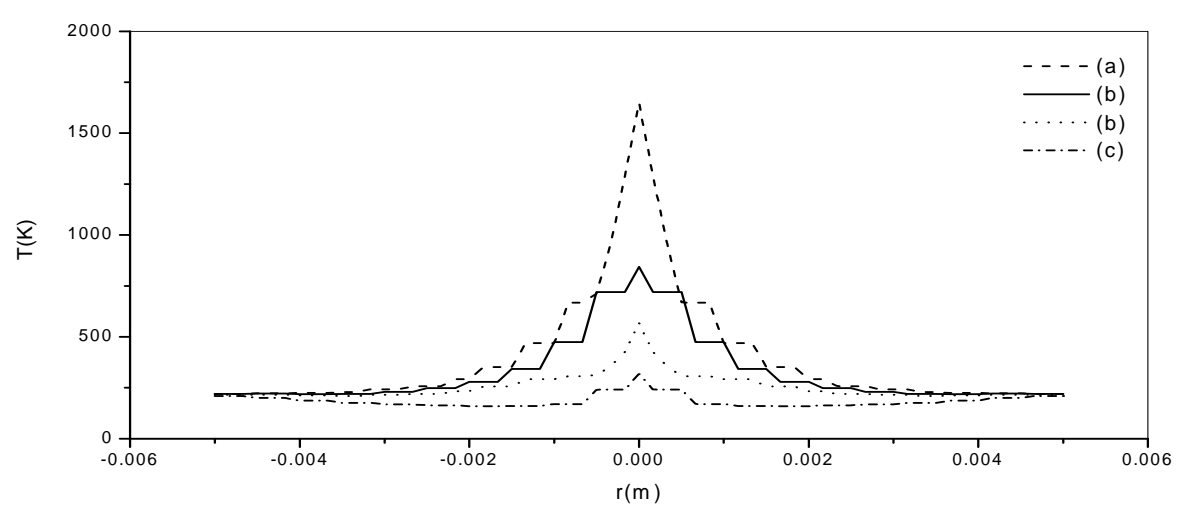

Fig. (2): The radial temperature distribution induced by the pump radiation calculated
at $\mathrm{t}=\tilde{\mathrm{t}}$ for (a) $\mathrm{R}_{\mathrm{o}}=10^{-4} \mathrm{~m}$,
(b) $\mathrm{R}_{\mathrm{o}}=5 \times 10^{-4} \mathrm{~m}$,
(c) $\mathrm{R}_{\circ}=10^{-3} \mathrm{~m}$ and
(d) $\mathrm{R}_{\mathrm{o}}=5 \times 10^{-3} \mathrm{~m}$.

\section{(b) Heating resulting from the laser beam. \\ (I) Gaussian distribution laser beam. \\ (i) $w_{\mathrm{o}}=10^{-4} \mathrm{~m}$}

Figure (3) represents the radial distribution of the net amplification coefficient calculated for $z=0$ at $t=\tilde{t}$ with $R_{o}$ as a parameter. The curves show that for all $R_{\circ}$ values a practically complete saturation of $\left(\beta_{L}-\alpha_{L}\right)$ occurs within $r=w_{o}$. For all $R_{o}$ values $\left(\beta_{L}-\alpha_{L}\right)$ attains the same behaviour and practically the same values as the corresponding ones of the linear case [17] after $r \cong w_{o}=10^{-4} \mathrm{~m}$.

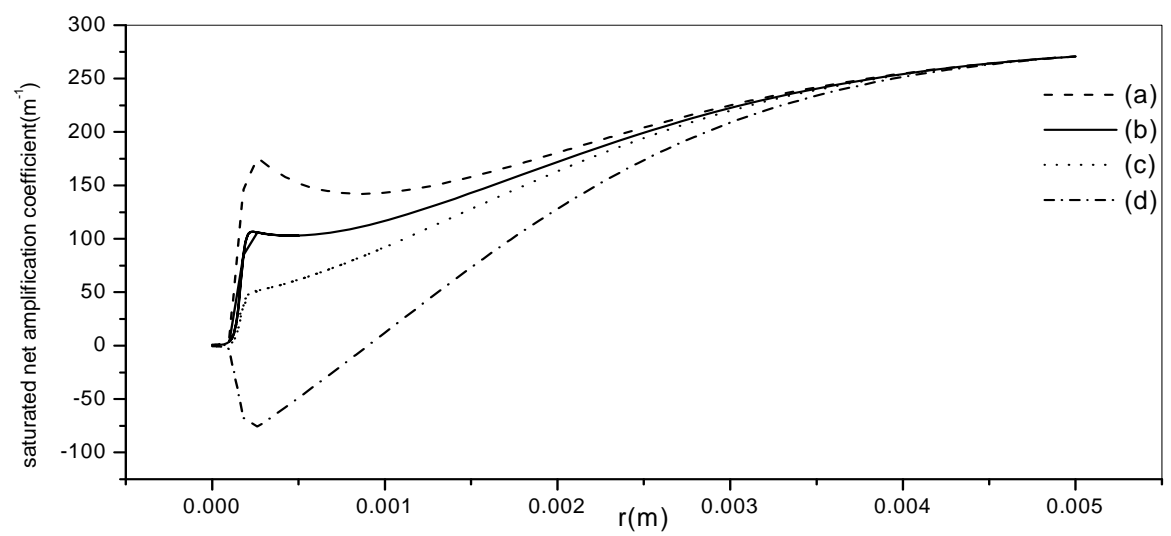

Fig. (3): The radial distribution of the saturated net amplification coefficient of the laser radiation having a Gaussian intensity distribution of width $w_{0}=10^{-4} \mathrm{~m}$ and pulse

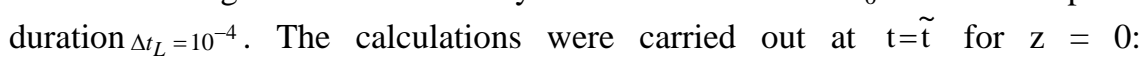
(a) $R_{0}=10^{-4} \mathrm{~m}$, (b) $R_{0}=5 \times 10^{-4} \mathrm{~m}$, (c) $R_{0}=10^{-3} \mathrm{~m}$ and (d) $R_{0}=5 \times 10^{-3} \mathrm{~m}$. 
Figure (4) represents the radial distribution of the net amplification coefficient calculated at $t=\tilde{t}$ for $z=L$ and $R_{o}$ as a parameter. Due to the fact seen from the calculation of the axial distribution, that in case of positive values of $\left(\beta_{L}-\alpha_{L}\right)$ in the vicinity of $r=0$ the radiation will be amplified as it propagate along the $z$ direction. This leads to brooding the range in which $\left(\beta_{L}-\alpha_{L}\right)$ is practically saturated. In case when $\left(\beta_{L}-\alpha_{L}\right)$ attains negative values the radiation will be practically total absorbed in the vicinity of $z=0$. Thus $\left(\beta_{L}-\alpha_{L}\right)$ for $z=L$ attain practically the same values as the corresponding ones of the linear case [17].

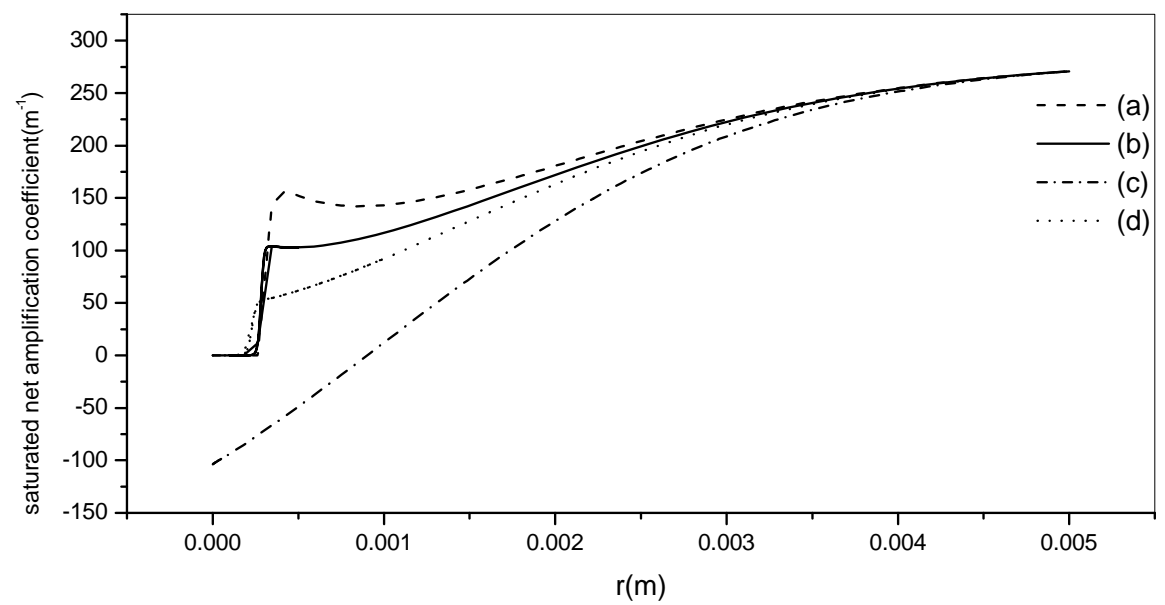

Fig. (4): The radial distribution of the saturated net amplification coefficient of the laser radiation having a Gaussian intensity distribution of width $w_{\circ}=10^{-4} \mathrm{~m}$ and pulse duration $\Delta t_{L}=10^{-4}$. The calculations were carried out at $\mathrm{t}=\tilde{\mathrm{t}}$ for $\mathrm{z}=\mathrm{L}$ : (a) $R_{0}=10^{-4} \mathrm{~m}$, (b) $R_{0}=5 \times 10^{-4} \mathrm{~m}$, (c) $\mathrm{R}_{\circ}=10^{-3} \mathrm{~m}$ and (d) $R_{0}=5 \times 10^{-3} \mathrm{~m}$.

Figure (5) represents the temporal temperature distribution calculated for $r=0$ and $z=0$ with $R_{o}$ as a parameter. The curves are independent of $R_{o}$ and exhibit the same behaviour and values as the linear case. This attribute can be explained by the fact that at $\mathrm{z}=0$ practically no absorption has taken place and that due to the bad conductivity and the small times at which the calculation was carried out the internal region has not affected the outer surface. 


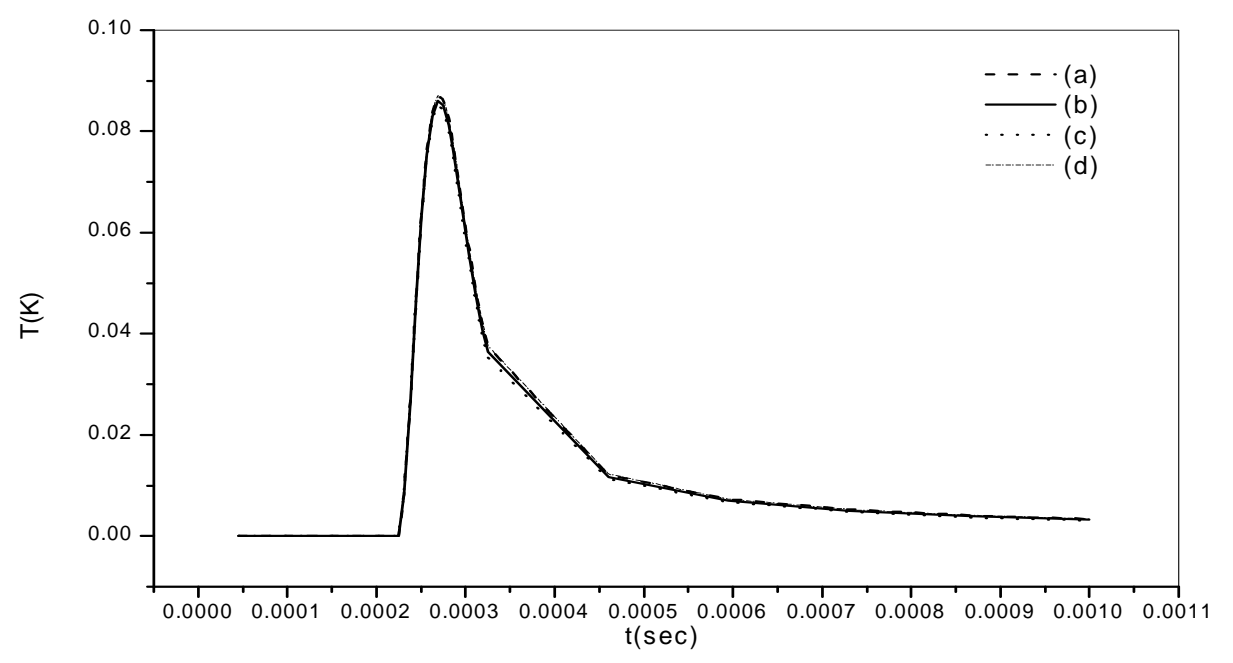

Fig. (5): The temporal temperature distribution induced by a laser beam having a Gaussian intensity distribution of $w_{\circ}=10^{-4} \mathrm{~m}$, and $\Delta t_{L}=10^{-4}$ considering saturation of amplification. The calculations were carried out for $\mathrm{z}=0$ and $\mathrm{r}=0$ : (a) $R_{\circ}=10^{-4} \mathrm{~m}$, (b) $R_{\circ}=5 \times 10^{-4} \mathrm{~m}$, (c) $R_{\circ}=10^{-3} \mathrm{~m}$ and (d) $R_{\circ}=5 \times 10^{-3} \mathrm{~m}$.

Figure (6) Represents the temporal temperature distribution calculated for $z=L$ and $r=0$ with $R_{o}$ as a parameter. From the curves it is evident that, the temperature attains its maximum value temporarily retarded from the maximum of the laser radiation. This behaviour can be attributed to the possibility that up to this time the stored heat energy is greater than the conducted one into the cooler part of the rod. The maximum of the temperature decreases with increasing $R_{o}$-values. This is due to the decreased saturated $\left(\beta_{L}-\alpha_{L}\right)$ with increasing $R_{o}$-values.

Figure (7) Represents the axial temperature distribution calculated for $r=0$ at $t=\tilde{t}$ with $R_{o}$ as a parameter. The curves up to $R_{\circ}=5 \times 10^{-4} \mathrm{~m}$ show a monotone increase of the temperature with increasing z-values. This behaviour is due to the rest amplification of the saturated net amplification coefficient (Figs. 3, 4) which leads to smaller temperature compared with the linear case [17]. 


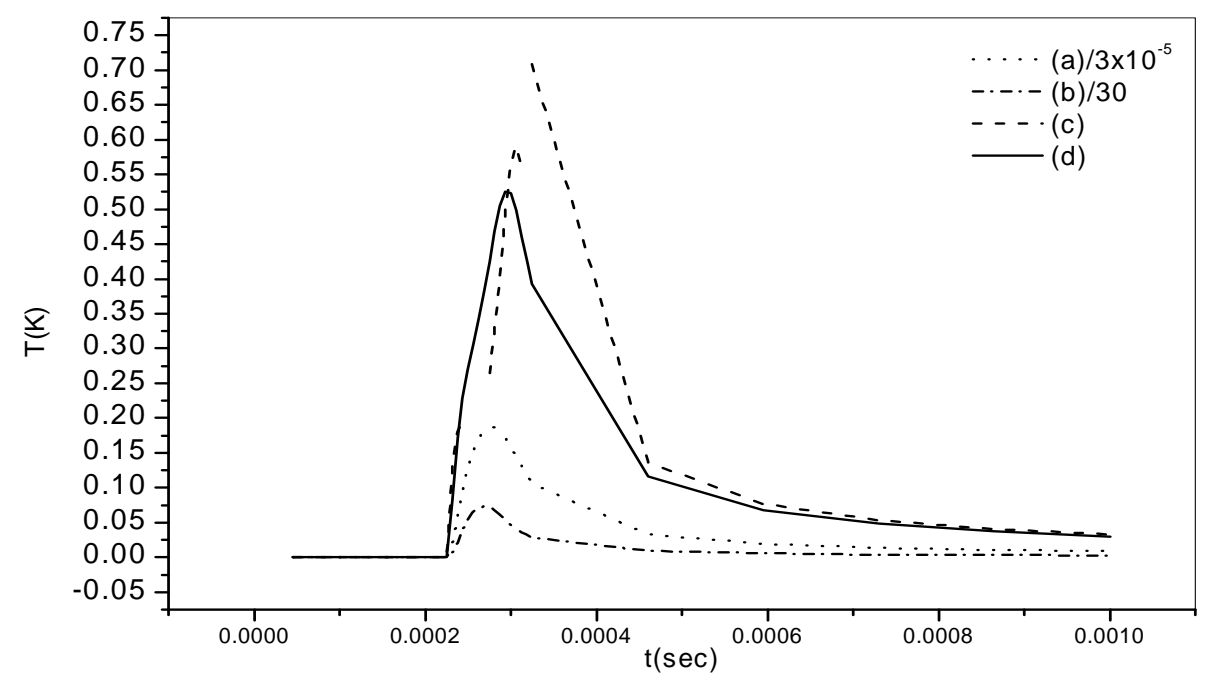

Fig. (6): The temporal temperature distribution induced by a laser beam having a Gaussian intensity distribution of $w_{\circ}=10^{-4} \mathrm{~m}$ and $\Delta t_{L}=10^{-4}$ considering saturation of amplification. The calculations were carried out for $\mathrm{z}=\mathrm{L}, \mathrm{r}=0$ : (a) $R_{\mathrm{o}}=10^{-4} \mathrm{~m}$, (b) $R_{\circ}=5 \times 10^{-4} \mathrm{~m}$, (c) $R_{\circ}=10^{-3} \mathrm{~m}$ and (d) $R_{\circ}=5 \times 10^{-3} \mathrm{~m}$.

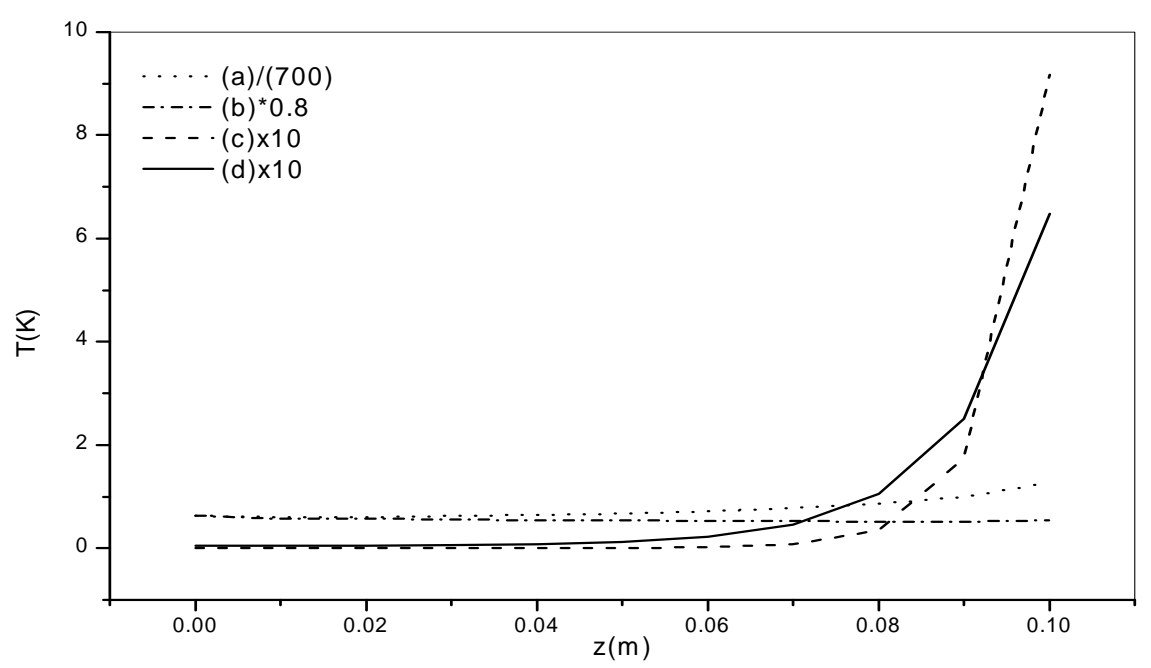

Fig. (7): The axial temperature distribution induced by a laser beam having a Gaussian intensity distribution of $w_{\circ}=10^{-4} \mathrm{~m}$ and $\Delta t_{L}=10^{-4}$ considering saturation of amplification. The calculations were carried out at $t=\tilde{t}$ for $\mathrm{r}=0$ : (a) $R_{\mathrm{o}}=10^{-4} \mathrm{~m}$, (b) $R_{\mathrm{o}}=5 \times 10^{-4} \mathrm{~m}$, (c) $R_{\mathrm{o}}=10^{-3} \mathrm{~m}$ and (d) $R_{\mathrm{o}}=5 \times 10^{-3} \mathrm{~m}$. 
For $R_{o}=10^{-3} \mathrm{~m}$ the curve exhibits a minimum shifts towards greater z-values than that for $R_{\circ}$ was considered the value $R_{o}=5 \times 10^{-3} \mathrm{~m}$. This behaviour can be explained as follows: For $R_{o}=10^{-3} \mathrm{~m},\left(\beta_{L}-\alpha_{L}\right)$ is positive, due to this fact the laser intensity will be amplified leading to reduction of for $\left(\beta_{L}-\alpha_{L}\right)$ and, thus, to the temperature decrease as $\mathrm{z}$ increases. This behaviour lasts up to the point at which the amplification of the mid-part of the laser radiation is strongly reduced and the wings become the upper hand which, due to their moderate intensity, does not saturate the amplification coefficient and thus the radiation will be amplified as $\mathrm{z}$ approach $(L)$. Due to this fact the temperature after the minimum increases monotonically. For $R_{o}=5 \times 10^{-3} \mathrm{~m}\left(\beta_{L}-\alpha_{L}\right)$ attains absorptive behaviour leading, due to the strong intensity of the laser radiation around $z=0$, to a strong reduction of $\left(\beta_{L}-\alpha_{L}\right)$ and thus to the strong negative slope around $z=0$. As $z$ approach values at which the intensity of the laser radiation is strongly reduced, the temperature decreases with smaller slope till $\mathrm{z}=0.09 \mathrm{~m}$, after which the wings of the laser radiation overcompensates the reduction in its mid-part intensity and the temperature increases slightly till $z=L$.

Figure (8) Represents the radial temperature distribution calculated at $t=\tilde{t}$ for $z=L$ and $R_{o}$ as a parameter. The figure shows a decrease of the maximum temperature at $r=0$ as $R_{o}$ increases. The explanation of this behaviour is the same as gives in figure (5-6). The appearance of a maximum at $r=0$ is due to the heating resulting at smaller times where saturation of the laser radiation has not taken place

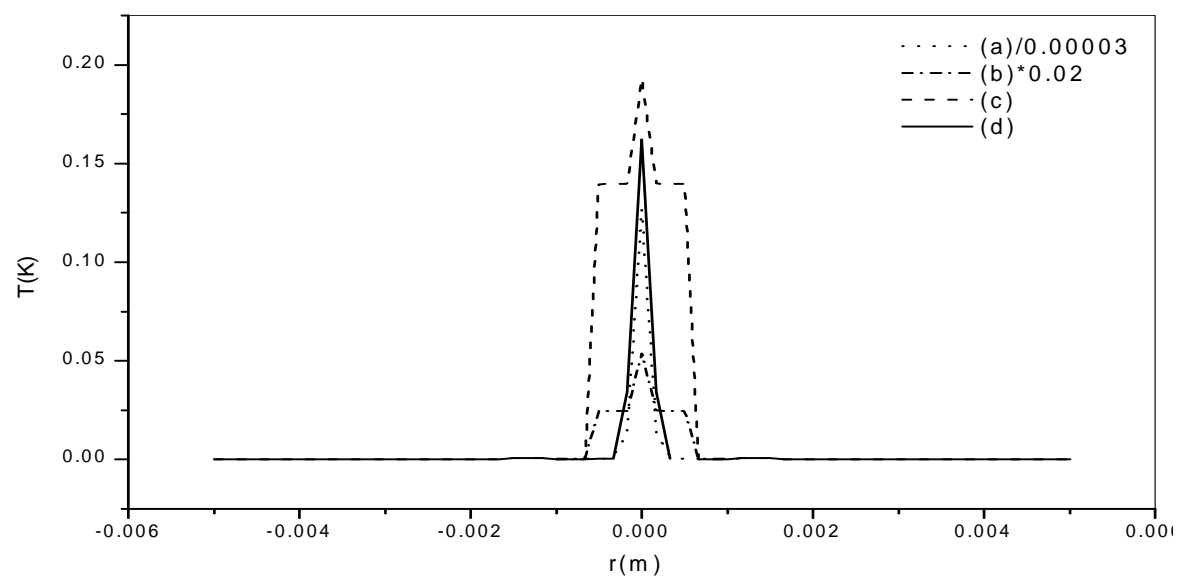

Fig. (8): The radial temperature distribution induced by a laser beam having a Gaussian intensity distribution of $w_{\circ}=10^{-4} \mathrm{~m}$ and $\Delta t_{L}=10^{-4}$ considering saturation of amplification. The calculations were carried out at $t=\tilde{t}$ for $\mathrm{z}=\mathrm{L}$ : (a) $R_{\circ}=10^{-4} \mathrm{~m}$, (b) $R_{\circ}=5 \times 10^{-4} \mathrm{~m}$, (c) $R_{\circ}=10^{-3} \mathrm{~m}$ and (d) $R_{\circ}=5 \times 10^{-3} \mathrm{~m}$. 
(ii) $w_{\circ}=5 \times 10^{-4} \mathrm{~m}$.

Figure (9) represents the radial distribution of the net amplification coefficient calculated at $t=\tilde{t}$ for $z=0$ and all $R_{o}$ values. Due to the greater half width of the laser radiation, the saturated interval around $r=0$ is greater than the corresponding ones represented in Fig. (3). The practically constance of the radial interval of the saturated amplification coefficient and its pronounced minima as $R_{o}$ increases can be explained in view of the corresponding curves obtained from the linear case [17].

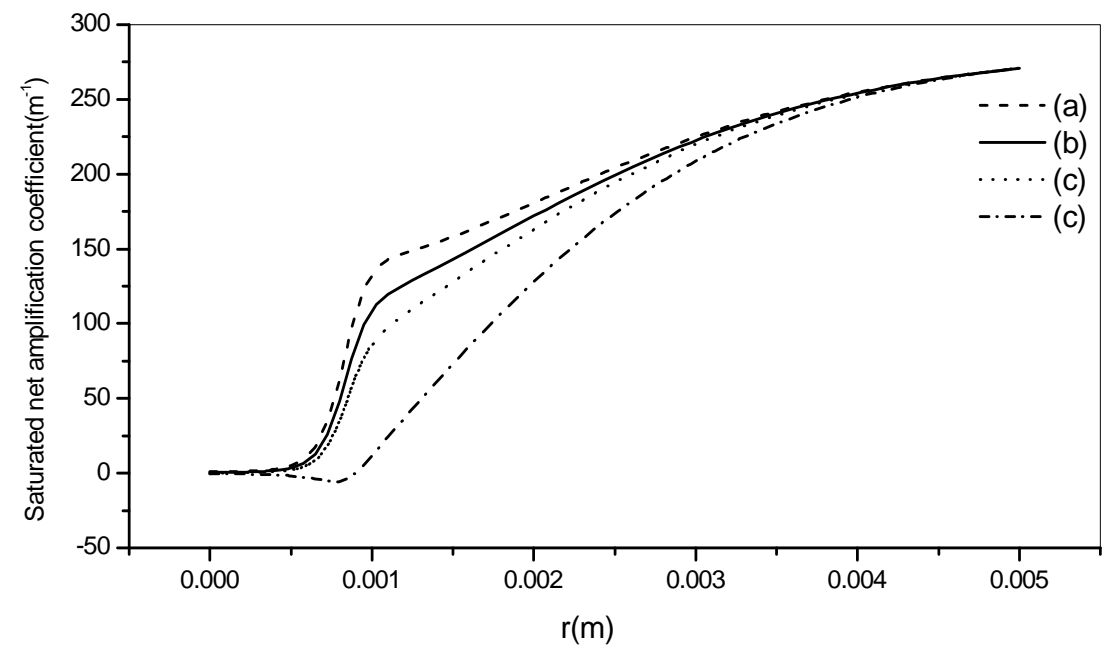

Fig. (9): The radial distribution of the saturated net amplification coefficient of the laser radiation having a Gaussian intensity distribution of width $w_{\circ}=5 \times 10^{-4} \mathrm{~m}$ and pulse duration $\Delta t_{L}=10^{-4}$. The calculations were carried out at $\mathrm{t}=\tilde{\mathrm{t}}$ for $\mathrm{z}=0$ : (a) $\mathrm{R}_{\mathrm{o}}=10^{-4} \mathrm{~m}$, (b) $\mathrm{R}_{\circ}=5 \times 10^{-4} \mathrm{~m}$, (c) $\mathrm{R}_{\mathrm{o}}=10^{-3} \mathrm{~m}$ and (d) $\mathrm{R}_{\mathrm{o}}=5 \times 10^{-3} \mathrm{~m}$.

Figure (10) represents the radial distribution of $\left(\beta_{L}-\alpha_{L}\right)$ calculated at $t=\frac{\Delta t_{p}}{4}$ for $z=L$ and all $R_{o}$ values. Due to the remaining amplification up to $R_{o}=10^{-3} \mathrm{~m}$ the range of saturation is wider than at $z=0$. In case where absorption occurs, the radiation is practically totally absorbed in the vicinity of $z=0$ leading to the appearance of the linear behavior [16].

Figure (11) represents the temporal temperature distribution calculated for $z=0, r=0$ and all considered $R_{o}$ values. Due to the reason in figure (5), the curves are independent of $R_{o}$ vand have the same values and behaviour of the temperature as the linear case. 


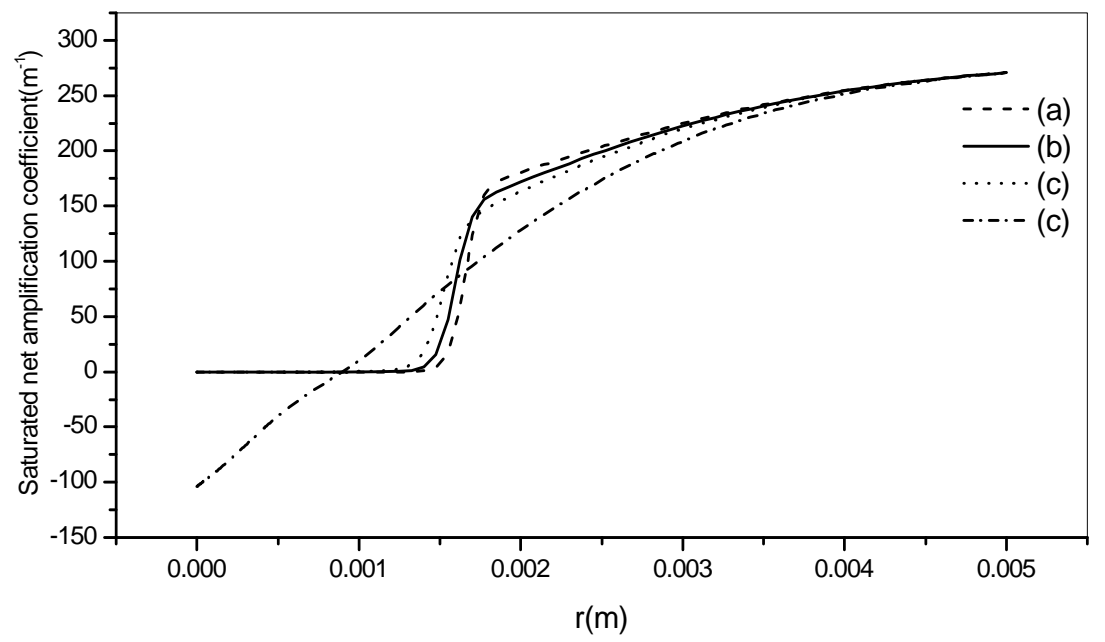

Fig. (10): The radial distribution of the saturated net amplification coefficient of the laser radiation having a Gaussian intensity distribution of width $w_{\circ}=5 \times 10^{-4} \mathrm{~m}$ and pulse duration $\Delta t_{L}=10^{-4}$. The calculations were carried out at $\mathrm{t}=\tilde{\mathrm{t}}$ for $\mathrm{z}=\mathrm{L}$ : (a) $\mathrm{R}_{\circ}=10^{-4} \mathrm{~m}$, (b) $\mathrm{R}_{\circ}=5 \times 10^{-4} \mathrm{~m}$, (c) $\mathrm{R}_{\circ}=10^{-3} \mathrm{~m}$ and (d) $\mathrm{R}_{\circ}=5 \times 10^{-3} \mathrm{~m}$.

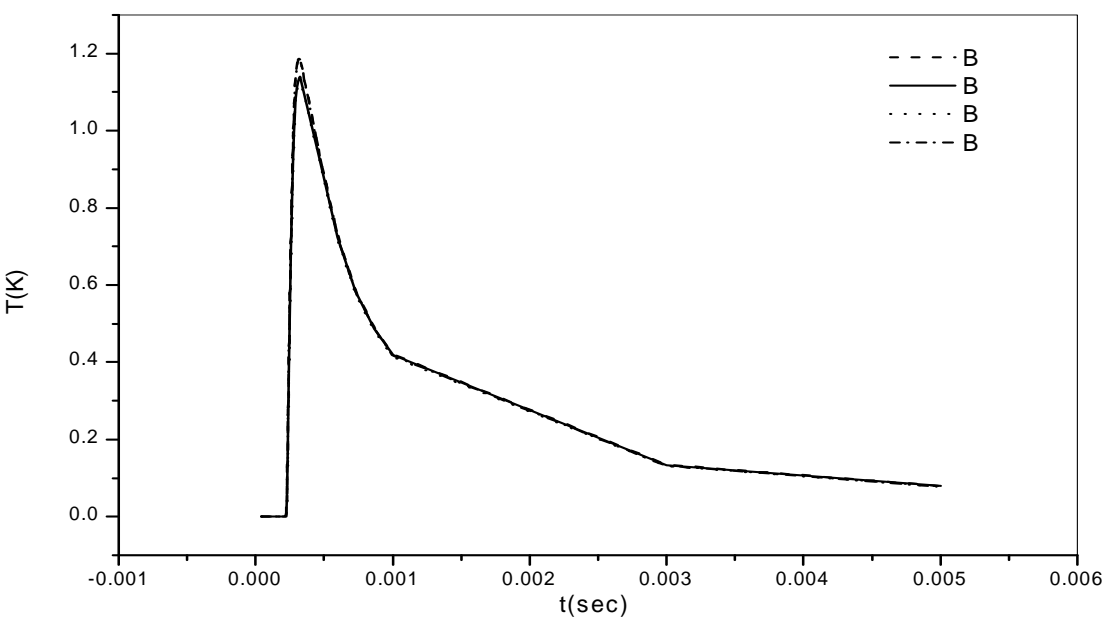

Fig. (11): The temporal temperature distribution induced by a laser beam having a Gaussian intensity distribution of $w_{\circ}=5 \times 10^{-4} \mathrm{~m}$, and $\Delta t_{L}=10^{-4}$ considering saturation of amplification calculated for $\mathrm{z}=0, \mathrm{r}=0$ : (a) $R_{\mathrm{o}}=10^{-4} \mathrm{~m}$, (b) $R_{\circ}=5 \times 10^{-4} \mathrm{~m}$, (c) $R_{\circ}=10^{-3} \mathrm{~m}$ and (d) $R_{\circ}=5 \times 10^{-3} \mathrm{~m}$.

Figure (12) represents the temporal temperature distribution calculated for $z=L, r=0$ and all considered $R_{o}$ values. The figure shows a smaller slope of the temperature after switching of the laser pulse resulting from the maxima 
shown in the radial distribution which leads to heat the axes of the rod. That the temperature attains much higher values for $R_{o}=10^{-4} \mathrm{~m}$ compared with the other $R_{o}$ values is due to the greater values of $\left(\beta_{L}-\alpha_{L}\right)$ in the surrounding of $r=0$ in this case than the other cases. The practically constant maximum of the temperature up to $R_{o}=5 \times 10^{-4} \mathrm{~m}$ is due to the behaviour of $\left(\beta_{L}-\alpha_{L}\right)$ which leads to practically the same absorption of the laser radiation in the vicinity of $z=0$.

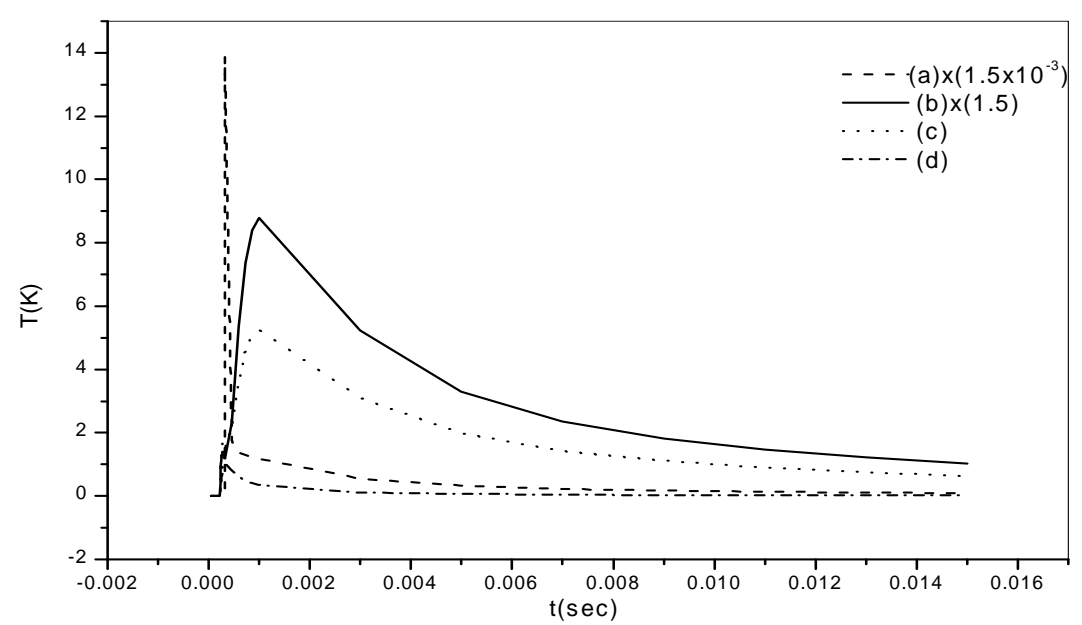

Fig.(12): The temporal temperature distribution induced by a laser beam having a Gaussian intensity distribution of $w_{\circ}=5 \times 10^{-4} \mathrm{~m}$ and $\Delta t_{L}=10^{-4}$ considering saturation of amplification calculated for $\mathrm{z}=\mathrm{L}, \mathrm{r}=0$ : (a) $R_{\circ}=10^{-4} \mathrm{~m}$, (b) $R_{\mathrm{o}}=5 \times 10^{-4} \mathrm{~m}$, (c) $R_{\mathrm{o}}=10^{-3} \mathrm{~m}$ and (d) $R_{\mathrm{o}}=5 \times 10^{-3} \mathrm{~m}$.

Figure (13) represents the axial temperature distribution calculated for $r=0$ at $t=\tilde{t}$ with $R_{o}$ as a parameter. Up to $R_{o}=10^{-3} \mathrm{~m}$ the figures show an increase of the temperature with increasing $z$ values. This behaviour is due to the overcompensation of the amplification coefficient. The maximum of the temperature attained at $z=L$ was found to decrease with increasing $R_{o}$ value. The explanation of this behaviour can be red from the radial behaviour of $\left(\beta_{L}-\alpha_{L}\right)$ which has the strongest slope for $R_{o}=10^{-4} \mathrm{~m}$ leading to an amplification of the radiation greater than the other $R_{o}$ values. As $R_{o}$ was given values greater than $10^{-3} \mathrm{~m}$ the temperature was found to decrease monotonically up to $z=0.09 \mathrm{~m}$ after which it begins to increase till $z=L$. This behaviour can be attributed to the strong absorption of the laser radiation which overcompensates the amplification. At $z=0.09 \mathrm{~m}$ the mid-part of the laser radiation was so strongly reduced that is don't markedly contribute to the temperature distribution. In this case the wings will be amplified leading to the small increase of the temperature in the vicinity of $z=L$. 


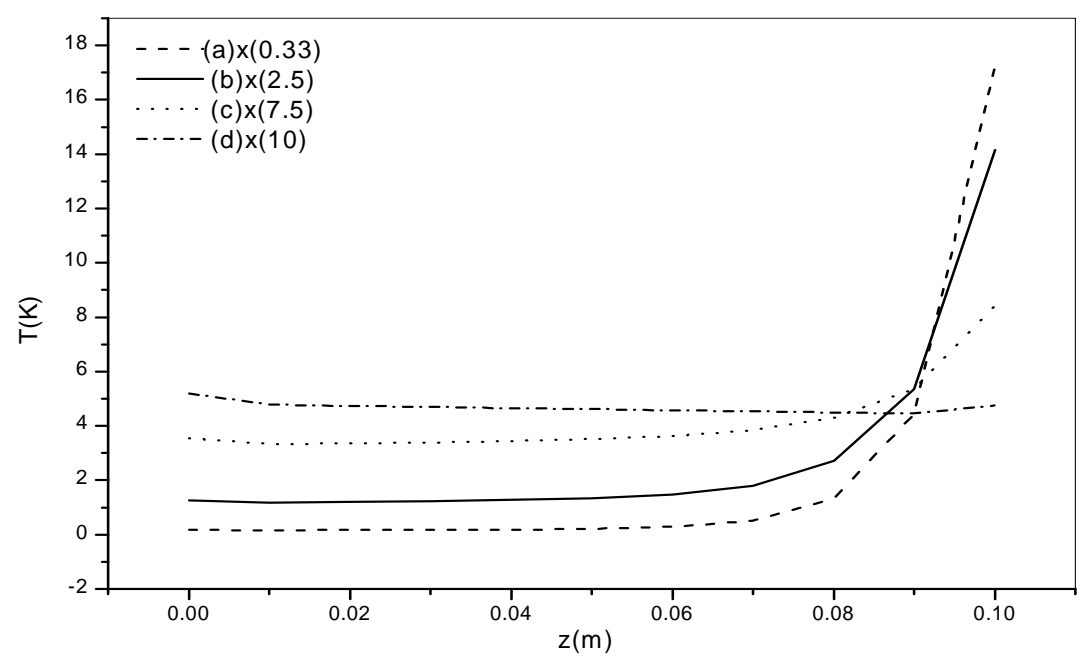

Fig. (13): The axial temperature distribution induced by a laser beam having a Gaussian intensity distribution of $w_{\circ}=5 \times 10^{-4} \mathrm{~m}$ and $\Delta t_{L}=10^{-4}$ considering saturation of amplification: (a) $R_{\circ}=10^{-4} \mathrm{~m}$, (b) $R_{\circ}=5 \times 10^{-4} \mathrm{~m}$, (c) $R_{\circ}=10^{-3} \mathrm{~m}$ and (d) $R_{\circ}=5 \times 10^{-3} \mathrm{~m}$. The calculations were carried out at $t=\tilde{t}$ for $\mathrm{r}=0$.

Figure (14) represents the radial distribution of the temperature calculated at $t=\tilde{t}$ for $z=L$ and $R_{o}$ as a parameter. The figure shows a dip up to $R_{o}=10^{-3} \mathrm{~m}$ this is due to the radial behaviour of $\left(\beta_{L}-\alpha_{L}\right)$ which is practically zero around $r=0$ after which it begins to increase leading to amplifying the wings of the laser radiation. Due to the stronger slope of $\left(\beta_{L}-\alpha_{L}\right)$ for $R_{o}=10^{-4}$ $\mathrm{m}$ than the other $R_{o}$ values the maximum of the temperature in this case is greater. For $R_{o}$ greater than $10^{-3} \mathrm{~m}$, the plateau of $\left(\beta_{L}-\alpha_{L}\right)$ is great enough such that the wings are not amplified enough to show a dip behaviour in the radial temperature profile.

(iii) $w_{\circ}=10^{-3} \mathbf{m}$.

Figure (15) represents the radial net amplification coefficient calculated at $t=\tilde{t}$ for $z=0$ and all considered $R_{o}$ values. Due to the greater half width of the laser radiation the radial interval of the saturated values is greater than the forgoing laser half widths. 


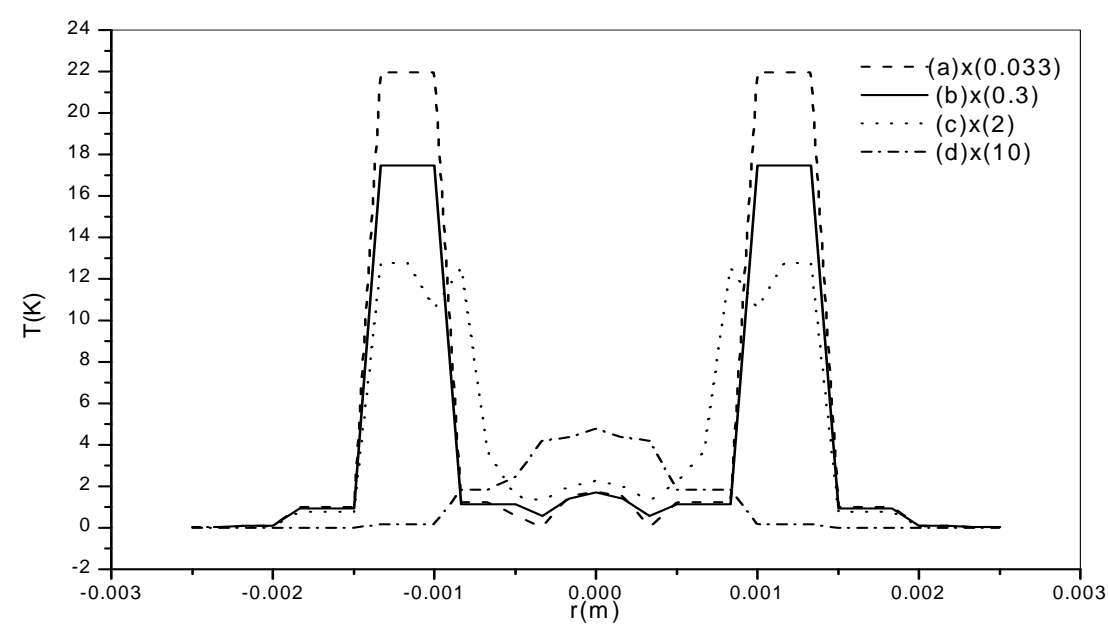

Fig. (14): The radial temperature distribution induced by a laser beam having a Gaussian intensity distribution of $w_{\circ}=5 \times 10^{-4} \mathrm{~m}$ and $\Delta t_{L}=10^{-4}$ considering saturation of amplification. The calculations were carried out at $t=\tilde{t}$ for $\mathrm{z}=\mathrm{L}$ and (a) $R_{\circ}=10^{-4} \mathrm{~m}$ (b) $R_{\circ}=5 \times 10^{-4} \mathrm{~m}$ (c) $R_{\circ}=10^{-3} \mathrm{~m}$ (d) $R_{\circ}=5 \times 10^{-3} \mathrm{~m}$.

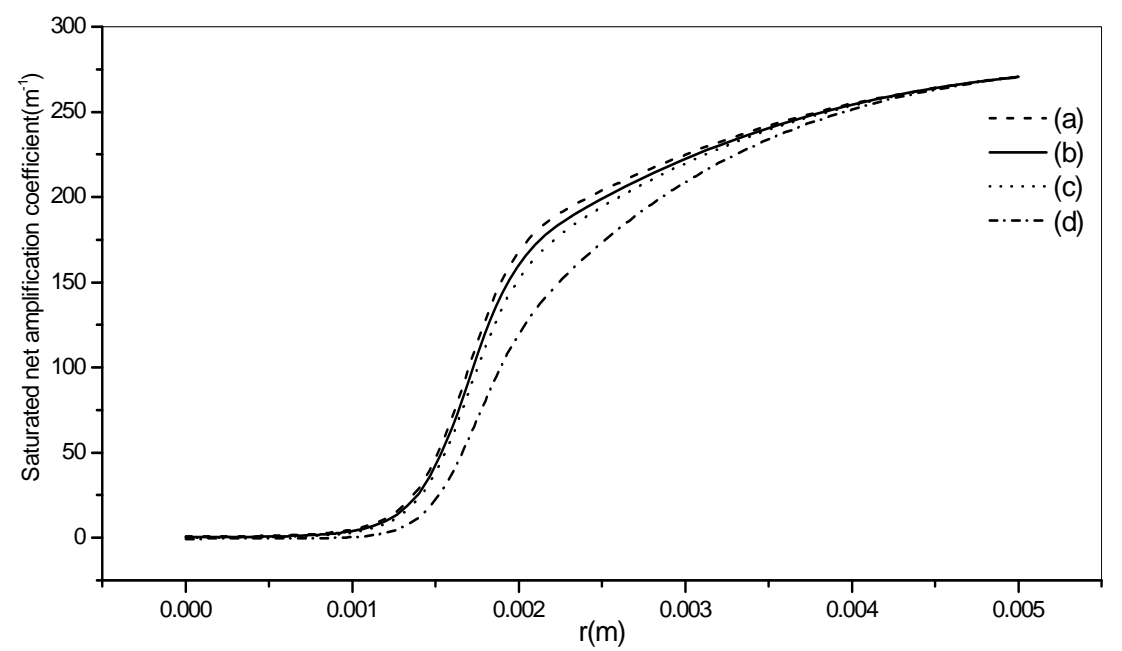

Fig. (15): The radial distribution of the saturated net amplification coefficient of the laser radiation having a Gaussian intensity distribution of width $w_{\circ}=10^{-3} \mathrm{~m}$ and pulse duration $\Delta t_{L}=10^{-4}$. The calculations were carried out at $\mathrm{t}=\tilde{\mathrm{t}}$ for $\mathrm{z}=0$ : (a) $\mathrm{R}_{\circ}=10^{-4} \mathrm{~m}$, (b) $\mathrm{R}_{\circ}=5 \times 10^{-4} \mathrm{~m}$, (c) $\mathrm{R}_{\circ}=10^{-3} \mathrm{~m}$ and (d) $\mathrm{R}_{\mathrm{o}}=5 \times 10^{-3} \mathrm{~m}$. 
Figure (16) represents the radial distribution of the net amplification $\left(\beta_{L}-\alpha_{L}\right)$ at $t=\tilde{t}$ for $z=L$ and $R_{o}$ as a parameter. Due to the great half width the range of the saturated values of $\left(\beta_{L}-\alpha_{L}\right)$ is greater than the corresponding ones in the case of smaller $w_{0}$-values. The step changing behaviour of the curves from $R_{o}=5 \times 10^{-3} \mathrm{~m}$ and upwards is due to the following, since practically all laser radiation was absorbed in the vicinity of $z=0$ the curves behaves as the linear case. Due to the amplification of the wings saturation takes place from $r=10^{-4} \mathrm{~m}$ to $3.5 \times 10^{-4} \mathrm{~m}$. After this interval the radiation of the laser is weak enough such that the linear behavior appears once more.

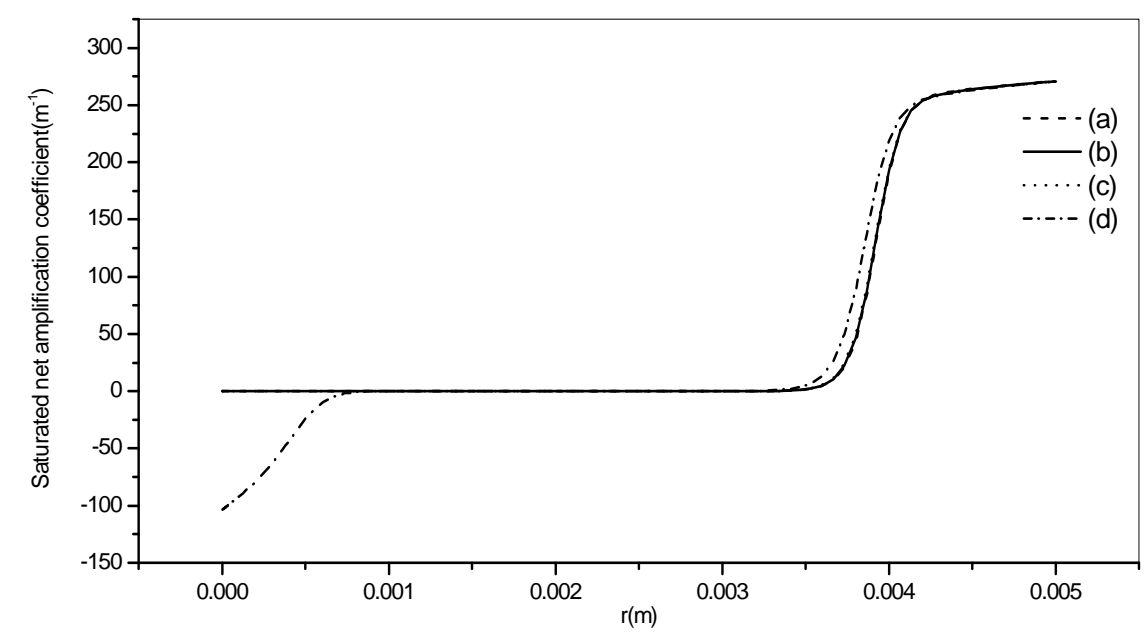

Fig. (16): The radial distribution of the saturated net amplification coefficient of the laser radiation having a Gaussian intensity distribution of width $w_{\circ}=10^{-3} \mathrm{~m}$ and pulse duration $\Delta t_{L}=10^{-4}$. The calculations were carried out at $\mathrm{t}=\tilde{\mathrm{t}}$ for $\mathrm{z}=\mathrm{L}$ : (a) $\mathrm{R}_{\mathrm{o}}=10^{-4} \mathrm{~m}, \quad$ (b) $\mathrm{R}_{\mathrm{o}}=5 \times 10^{-4} \mathrm{~m}, \quad$ (c) $\mathrm{R}_{\mathrm{o}}=10^{-3} \mathrm{~m}$ and (d) $\mathrm{R}_{\mathrm{o}}=5 \times 10^{-3} \mathrm{~m}$.

Figure (17) represents the temporal temperature distribution calculated for $z=0, r=0$ and all considered $R_{o}$ values. The curves show the same values and behaviour as the case of $w_{0}=10^{-3} \mathrm{~m}$ in the linear case; this is due to the same reason in Figs. $(5,11)$. 


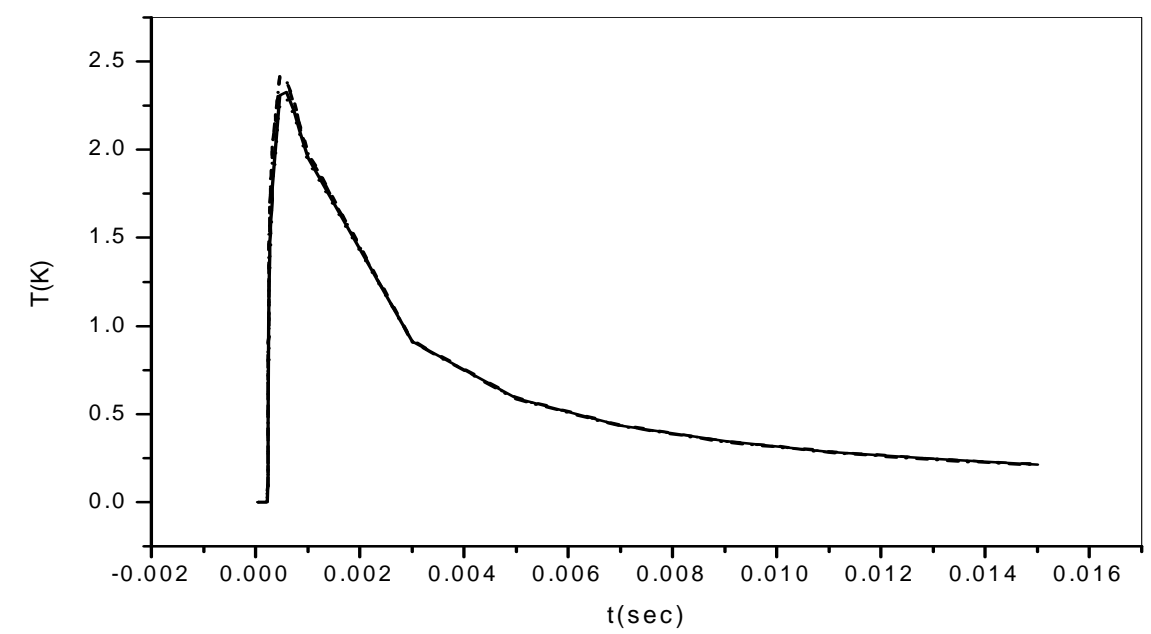

Fig. (17): The temporal temperature distribution induced by a laser beam having a Gaussian intensity distribution of $w_{\circ}=10^{-3} \mathrm{~m}$ and $\Delta t_{L}=10^{-4}$ considering saturation of amplification. The calculations were carried out for $\mathrm{z}=0$ and $\mathrm{r}=0$ : (a) $R_{\circ}=10^{-4} \mathrm{~m}$, (b) $R_{\circ}=5 \times 10^{-4} \mathrm{~m}$, (c) $R_{\circ}=10^{-3} \mathrm{~m}$ and (d) $R_{\circ}=5 \times 10^{-3} \mathrm{~m}$.

Figure (18) represents the temporal temperature distribution calculated for $z=L, r=0$ with $R_{o}$ as a parameter. It shows that the maximum temperature decreases with increasing $R_{o}$ values is due to the greater amplification coefficient before reaching the saturation with increasing $R_{0}$. After switching of the laser pulse the temperature begins to decrease due to conduction of heat in the cooled parts around $r=0$.After a while the heat diffusion from the maxima in the radial distribution to reach the axes of the rod and increases its temperature leading to a second relative maxima.

Figure (19) represents the axial temperature distribution calculated at $t=\tilde{t}$ for $r=0$ and $R_{o}$ as a parameter. All curves show a monotone increase of different slopes with increasing $z$ values. This behaviour is because of the great half width of the laser radiation which attains positive values of $\left(\beta_{L}-\alpha_{L}\right)$ at smaller time and in its wings. The decrease in the slope with increasing $R_{o}$ values is due to the smaller amplification of $\left(\beta_{L}-\alpha_{L}\right)$ for great $R_{o}$ values. 


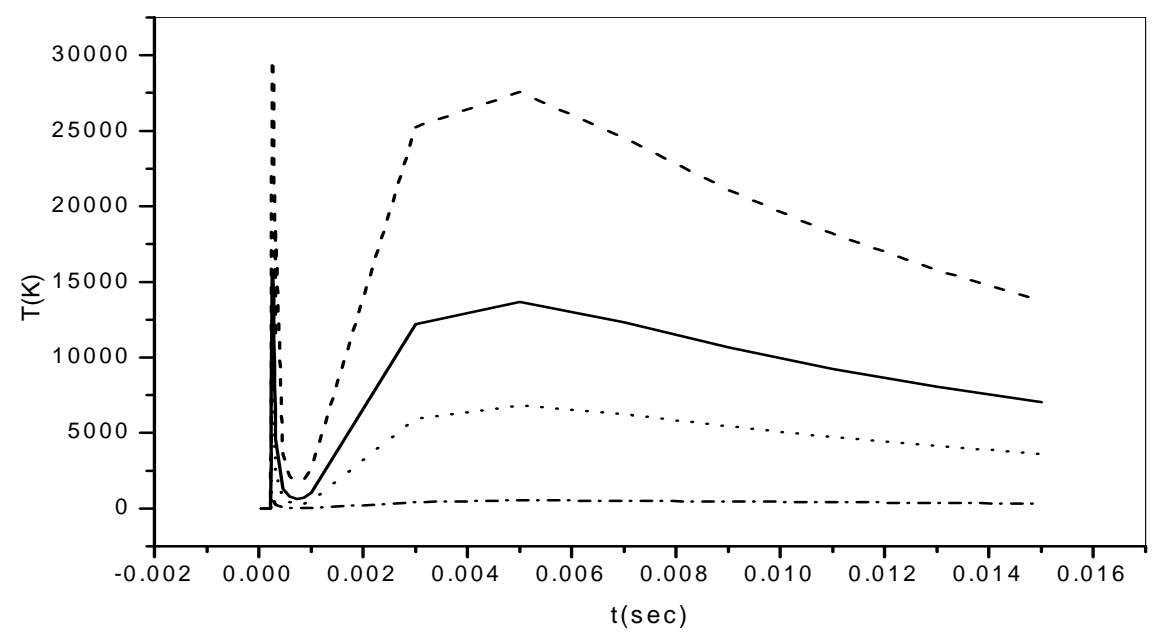

Fig. (18): The temporal temperature distribution induced by a laser beam having a Gaussian intensity distribution of $w_{\circ}=10^{-3} \mathrm{~m}$ and $\Delta t_{L}=10^{-4}$ considering saturation of amplification. The calculations were carried out for $\mathrm{z}=\mathrm{L}, \mathrm{r}=0$ : (a) $R_{\circ}=10^{-4} \mathrm{~m}$, (b) $R_{\circ}=5 \times 10^{-4} \mathrm{~m}$, (c) $R_{\circ}=10^{-3} \mathrm{~m}$ (d) $R_{\circ}=5 \times 10^{-3} \mathrm{~m}$.

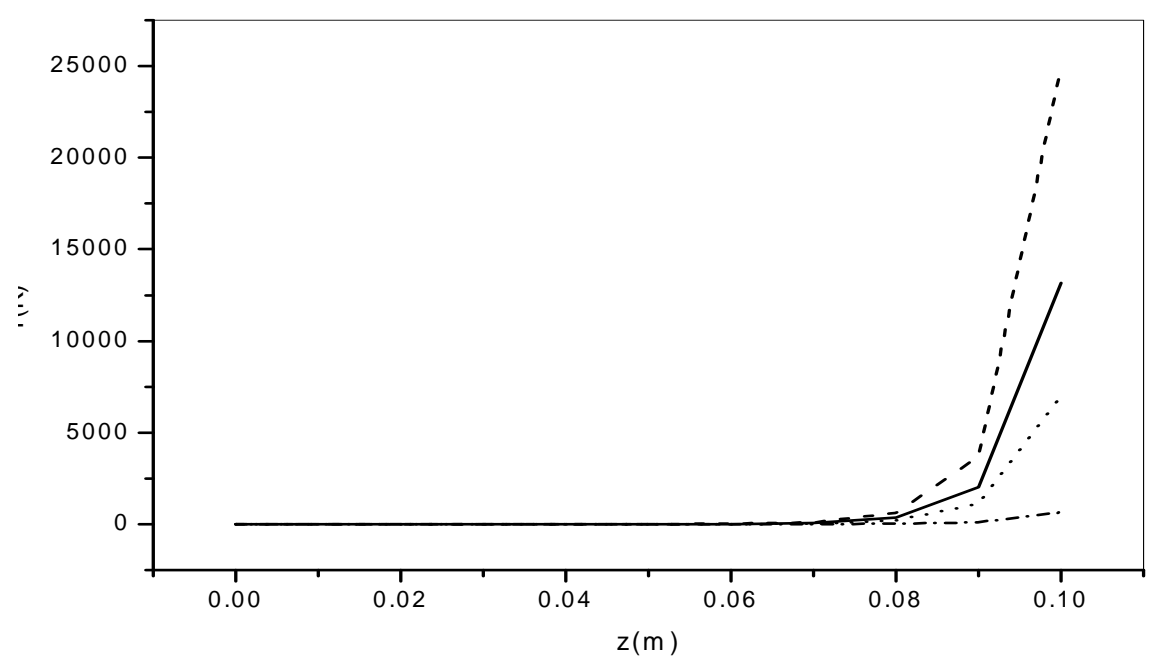

Fig. (19): The axial temperature distribution induced by a laser beam having a Gaussian intensity distribution of $w_{\circ}=10^{-3} \mathrm{~m}$ and $\Delta t_{L}=10^{-4}$ considering saturation of amplification. The calculations were carried out at $t=\tilde{t}$ for $\mathrm{r}=0$ : (a) $R_{\circ}=10^{-4} \mathrm{~m}$, (b) $R_{\circ}=5 \times 10^{-4} \mathrm{~m}$, (c) $R_{\circ}=10^{-3} \mathrm{~m}$ and (d) $R_{\circ}=5 \times 10^{-3} \mathrm{~m}$. 
Figure (20) represents the redial temperature distribution at $t=\tilde{t}$ for $z=L$ and $R_{o}$ as a parameter. Due to the broader half width of the laser radiation, its wings will be more amplified than the central part during the onset of saturation; this behaviour leads to the appearance of the dip. The decrease of the maximum temperature with increasing $R_{o}$ values can be explained in view of the $\left(\beta_{L}-\alpha_{L}\right)$ profile which has great amplification values before and during reaching saturation as $R_{o}$ is assigned small values. The explanation of appearance of the maximum at $r=0$ is given in Figure (8).

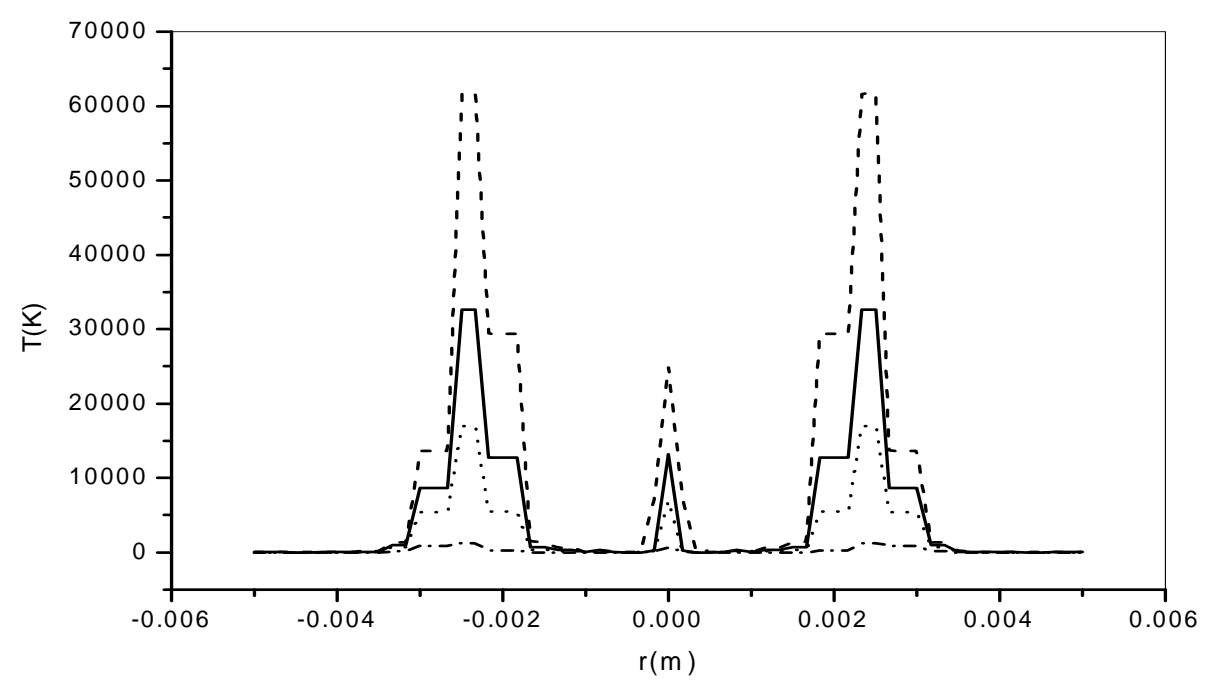

Fig. (20): The radial temperature distribution induced by a laser beam having a Gaussian intensity distribution of $w_{\circ}=10^{-3} \mathrm{~m}$ and $\Delta t_{L}=10^{-4}$ considering saturation of amplification. The calculations were carried out at $t=\tilde{t}$ for $\mathrm{z}=\mathrm{L}$ : (a) $R_{\circ}=10^{-4} \mathrm{~m}$, (b) $R_{\mathrm{o}}=5 \times 10^{-4} \mathrm{~m}$, (c) $R_{\mathrm{o}}=10^{-3} \mathrm{~m}$ and (d) $R_{\mathrm{o}}=5 \times 10^{-3} \mathrm{~m}$.

\section{Conclusion:}

The net amplification coefficient calculated for $r=0$ at $\tilde{t}$ was found to decrease with increasing $z$ values. At $z=L$ the medium is practically neither amplifying nor absorbing where it was amplifying in the linear case. For r-values greater than the half width of the laser beam, the net amplification coefficient tends to the linear behaviour. The calculated radial distribution of $\left(\beta_{L}-\alpha_{L}\right)$ at $\tilde{t}$ for $z=0$ shows that, the width of the saturation behaviour around $r=0$ increases with increasing $w_{\circ}$ values after which it behaves as the linear case. At $z=L$ the saturated interval was found to increase with increasing $w_{\circ}$ values only for the case where $\left(\beta_{L}-\alpha_{L}\right)$ was positive in the linear case. As 
$\left(\beta_{L}-\alpha_{L}\right)$ was absorptive in the linear case, the strong absorption in the vicinity of $\mathrm{z}=0$ leads to the linear behaviour of $\left(\beta_{L}-\alpha_{L}\right)$ at $\mathrm{z}=L$. As the wings was amplified enough, saturation of $\left(\beta_{L}-\alpha_{L}\right)$ takes place. The calculated temporal temperature distribution for $z=0$ and $r=0$ was found to be practically equal to that of the linear case. In case where saturation of $\left(\beta_{L}-\alpha_{L}\right)$ occurs at $z=L$, the temperature was found to be smaller than the linear case. Where a dip was found in the radial temperature distribution, a secondary maxima was found in the temporal temperature distribution calculated at $z=L$ and $r=0$.

\section{References:}

1. E. M. Breinan, B. H. Kear and C. M. Banas, Processing Materials with Laser Phys. Today 29, 44 (1967).

2. S. S., Charaschan "Laser Industry", Van Nostrand, New York, Ch. 4 and 5 (1972).

3. J. F. Ready "Effects of High Power Laser Radiation", Acadimic press, New York (1971).

4. M. Van Elsen, M. Baelmans, P. Mercelis and J. P. Krut, International Journal of Heat and Mass Transfer, 50, 4872 (2007).

5. T. Kurita, K. Komatsuzaki and M.Hattori, International Journal of Machine Tools \& Manufacture, 48, 220 (2008).

6. J. Jiao and X. Wang, Optics \& Laser Technology, 40, 297 (2008).

7. M. Sabaeian and H. Nadgaran, Optics Comunications, 281, 672 (2008).

8. J. F. Ready, J. Appl. Phy. 36, 426 (1965).

9. F. E. Harper, and M. I. Cohen, Solid State Electronics 11, 1176 (1968).

10. J. Jabczynski, K. Kopczynski and A. Szczesniak, Opt Eng. 35 (12), 3572 (1996).

11. C. Pfistner, R. Weber, HP. Weber, S. Merrazzi and R.Gruber, IEEE J. Quantum Electron, 30, 1605 (1994).

12. P. Marcello, G. Agostino and G. P. Anna, Solid-State Electronics 45, 511 (2001).

13. A. Hassan, M. M. Elniklawy, M. K. El-Adawi, E. M. Nasr, A. A. Hemida and O. A. Abd El-Gaffar, Optics and Laser Technology 28 (5), 337 (1996).

14. M. Necatiozisil "Heat Conduction", John Wiley \& Sonsinc (1993).

15. W. Koechner, "Solid State Laser Engineering", $4^{\text {th }}$ ed. Berlin Springer, (1992).

16. R. Zurmuehl, "Praktich Mathamatik Fuer Ingenieure und Physiker", Springer-Velag, Berlin (1965).

17. M. El-Nicklawy, A. Hassan and A. Abd-Rabu, Egypt. J. Solids 30 (2), 219 (2007). 
